\title{
FORMATION MECHANISMS OF THE SPLIT TAIL OF COMET BRADFIELD 1987XXIX
}

\author{
Ken Sugawara \\ Atsugi City Children's Science Center \\ 1-1-3, Nakachou, Atsugi-shi, Kanagawa 243, Japan \\ and \\ Jun-ichi Watanabe \\ National Astronomical Observatory \\ Mitaka, Tokyo, 181, Japan
}

\begin{abstract}
Comet Bradfield 1987XXIX showed a well-developed split tail, which is a dust tail feature divided into two branches by a dark gap. Similar structures have been observed in several comets. The split tail of comet Seki-Lines is one of them; some authors tried to explain it by rapid dust vaporization or self-regulation model. However, these models can not explain in the case of comet Bradfield 1987s. It was suggested in our previous works that the trend of variation of position angle of the dark gap coincides well with that of the plasma tail or projected radial vector. We discuss the formation mechanisms of the split tail from the view point of dust-plasma interactions.
\end{abstract}

\section{Introduction}

Comet Bradfield $1987 \times \times I X$ became a naked-eye object during a few months around the perihelion passage. Many photographs also show the existence of dark gap(hereafter DG) in the dust tail. The dust tail was divided into two branches by DG. This structure has been called a split tail, which has been observed in several comets. However, comet Seki-Lines is the only example analyzed for formation mechanisms(Jambor, 1973). In this paper, the morphological characteristics of this structure are reported, and probable formation mechanisms are discussed.

\section{Observational Material and Analysis}

Photographs of comet Bradfield taken from late Sep.1987 to late Feb. 1988 by many amateur astronomers in Japan were examined in order to know the morphology of the dust tail. We found DG in most of the photographs taken after late 0ct. 1987. These structures are dust tail features, because the DG exists on the plates taken in red spectral region(Watanabe et al., 1989).

For quantitative analysis of the morphology, the dust tail was fitted by calculated synchrone and syndyne curves with the Bessel-Bredikhin model, which is a mechanical theory based on the orbital motion of dust particles. Figs. 1 and 2 show the time variation of the position angle

A.C. Levasseur-Regourd and H. Hasegawa (eds.), Origin and Evolution of Interplanetary Dust, 273-276. (C) 1991 Kluwer Academic Publishers, Printed in Japan. 
for DG, plasma tail and synchrone curves(the locus of particles emitted from the nucleus at the same time), respectively. These show the location of DG near the plasma tail. The variation of position angle of DG coincides well with that of the plasma tail or projected radial vector rather than that of synchrones.

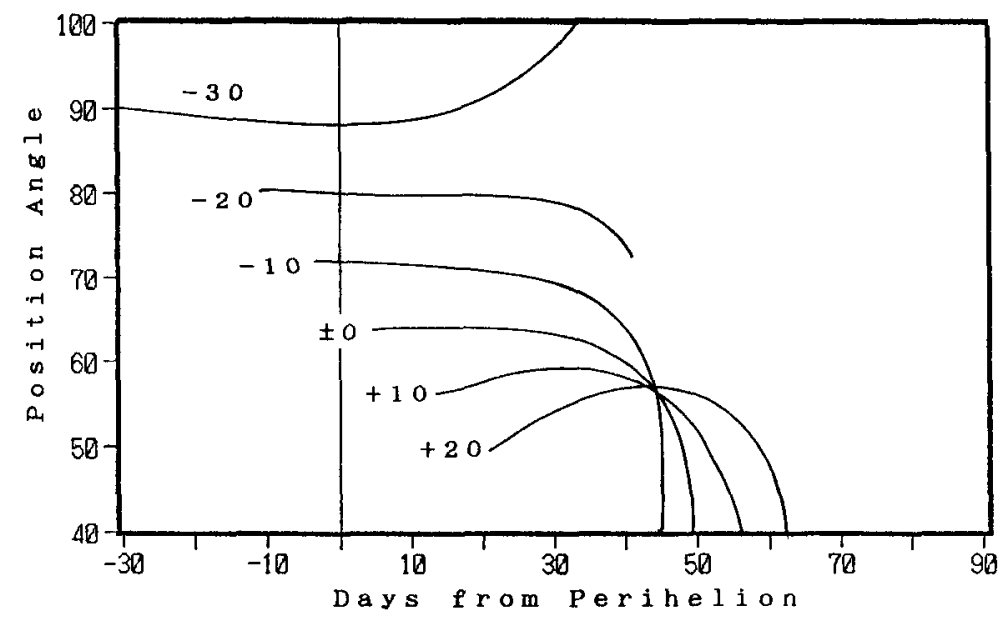

Fig. 1. Time (measured as the elapsed time from periherion passage) variation of the position angle(measured counterclockwise from the North) of dark gap(dots or vertical lines), plasma tail(cross marks), and projected radial vector(solid line).

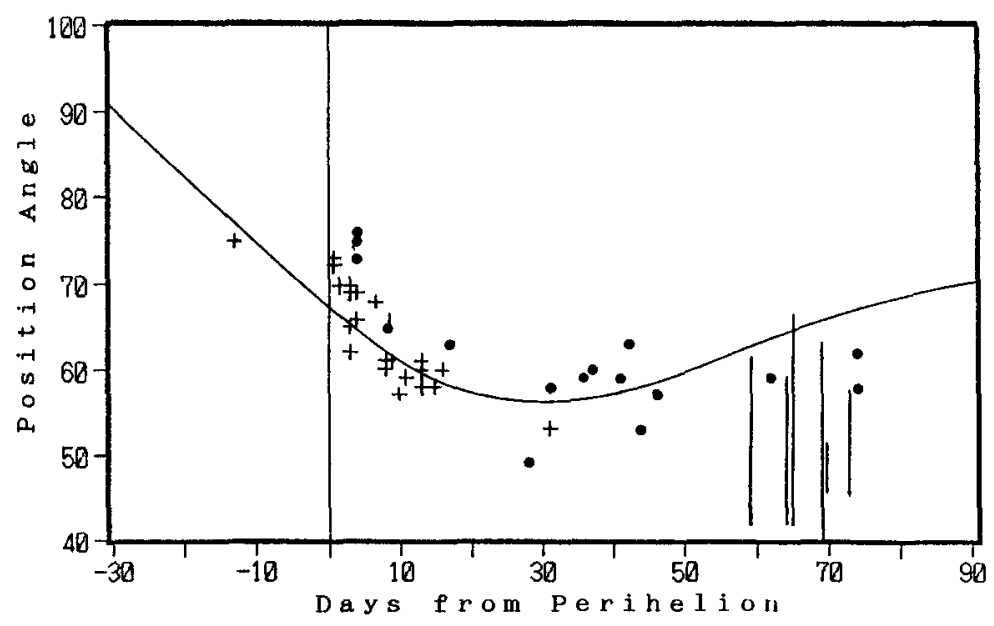

Fig. 2. Time variation of the position angle of initial directions of synchrones. Noted numbers correspond to respective times (interval to perihelion passage) of ejection.

\section{Discussion}




\subsection{RAPID VAPORIZATION MODEL OF DUST PARTICLES}

The position of the DG in Seki-Lines coincided with the synchrone for dust particles emitted at perihelion(Jabor, 1973). Jambor suggested that evaporation of dust emitted at perihelion(q=0.03 a.u.) could be the cause of the DG, although Sekanina(1976) noted that it was impossible to explain a DG in this way, unless the particles emitted at perihelion were different in composition(i,e. more volatile) than the particles in the dust tail which were emitted earlier. At the perihelion distance of comet Bradfield ( $q=0.8$ a.u.), however, the dust vaporization will be negligible, and the rapid vaporization model can be ruled out.

\subsection{SELF-REGULATION MODEL}

The self-regulation model suggested by Sekanina(1976) is as follows. A sharp peak in the dust production rate near perihelion caused a high opacity for solar radiation, reducing the solar energy impinging on the cometary nucleus.

If the optical depth of the cometary coma suddenly increases by an outburst, a lot of dust particles are required. Then a clear synchrone feature should appear in the dust tail. However, corresponding synchrone structure was not observed. This model also requires that the direction of DG coincides with the synchrone curve at perihelion, contrary to the observational data. Therefore, it is difficult to explain the formation of the split tail of this comet by the self-regulation model.

\subsection{RELATION BETWEEN PLASMA TAIL AND DUST TAIL}

The direction of DG coincides with that of the projected radial vector(or plasma tail) rather than that of synchrone around perihelion. It is known that an electric current flows along the tail axis at any time. Thus, there is a possibility that DG was formed by electromagnetic effects. Two models are following.

3.3.1. Sweeping Model. The broad tail extending from the head consists of relatively small dust particles, on which the repulsive force by the solar radiation is large. The electrostatic charging effect is also larger with smaller particles. Interaction of the cometary dust with the solar wind was studied by several authors(e.g. Ip et al. ; 1985). They concluded that the Lorentz force for the charged dust particle was important. The magnetosphere of comets has been revealed by in-situ observations, which have confirmed the existence of strong magnetic and electronic fields in the vicinity of the plasma tail axis. Hence the Lorenz force on dust particles would be more important near the axis than far from the tail axis. The Lorentz force could rapidly accelerate the dust particles in the current sheet, creating a void which is seen as a dark gap.

3.3.2. Electrostatic Disruption Model. Rhee(1976) showed that fluffy 
dust particles of micron or submicron sizes will be blown up if their surface potential reached a certain value. Following Ip(1984), the critical size for electrostatic disruption of spherical dust particles is

$$
\mathrm{R} \circ(\mu \mathrm{m})=9.4|\Phi| / \mathrm{F}_{t^{1 / 2}}
$$

where $R_{c}$ is critical size, $F_{t}$ is tensile strength of dust particle, $\Phi$ is surface potential[V]. Following Burns et al.(1979), we convert $R$ 。 into $\beta$ (ratio of the solar gravitation to solar radiation pressure) in the case of the Mie scattering efficeincy for radiation pressure is 1 and the density of dust particle(assumed as sphelical) is $1\left[\mathrm{~g} / \mathrm{cm}^{3}\right]$. Following Rhee (1976), we assumed $F_{t}=10^{4}\left[\right.$ dyne $\left./ \mathrm{cm}^{2}\right]$. If $\beta=0.5$, dust particles disrupt into submicron sizes when $|\Phi| \sim 10$. The $\beta$ value of $D G$ derived from comparing Bessel-Bredikhin theory with observations is approximately $<0.5$. Judging from model calculations(Hill and Mendis;1980), and previous discussion, it is suggested that DG formed by electrostatic disruption if the surface potential $\Phi$ reached sufficiently high value.

In order to conclude what is most important in the effects of electrostatic disruption, sweeping, or combination of them, more work would be necessary.

\section{Acknowledgements}

We express our thanks to many Japanese amateur astronomers, especially Messrs. M. Tsumura, S. Mizuno, K. Osada and S. Yamane, H. Akisawa, T. Oka, and $\mathrm{H}$. Hasegawa. We are also thankful to the editors of Gekkan-Tenmon, of Tenmon-Gaido and of sky Watcher for their permission to measure the published and non-publishd photographs.

\section{References}

Burns, J.A., Lamy, P.L., and Soter, S. (1979)' Radiation Forces on Small Particles in the Solar System.' ICARUS, 40, 1-48.

Hill, J.R. and Mendis,D.A. (1980)' On the Origin of Striae in Cometary Dust Tails.' Astrophys. J., 242, 395-401.

Ip., W.-H., (1984) 'Comet-Solar Wind Interactions:A Dusty Point of View.' Adv.Space Res. Vol.4, No.9, 239-247.

Ip., W.-H., Kimura, H., and Liu, C.-P. (1985)' Interaction of the Cometary Dust with the Solar Wind and Cometary plasma.' in Properties and Interactions of Interplanetary Dust, edt. R.H.Giese and P. Lamy (D. Reidel), 325-328.

Jambor, B.J.(1973)' The Split Tail of Comet Seki-Lines.' Astrophys. J., 185, 727-734.

Rhee,J.W.(1976)' Electrostatic Disruption of Lunar Dust Particles.' in Interplanetary Dust and Zodiacal Light,p.238

Sekanina, Z.(1976) , Progress in Our Understanding of Cometary Dust Tails.' in The Study of Comets, pt.2, 893-939.

Watanabe, J., Aoki, T., Taniguchi, Y., and Tarusawa, K. (1989) 'Photographic Observations of Comet Bradfield 1987XXIX at the Kiso Observatory.' Publ. Natl. Astron. Obs. Japan, 7, 71-84. 